\title{
INTRINSIC KINEMATICS OF MOVING COMPONENTS IN CURVED RELATIVISTIC JETS
}

\author{
L. LARA ${ }^{1,2}$, A. ALBERDI ${ }^{2,3}$ \\ 1 IRA - CNR, Via Gobetti 101, 40129 Bologna, Italy \\ ${ }^{2}$ IAA - CSIC, Apdo. 3004, 18080 Granada, Spain \\ ${ }^{3}$ LAEFF, Apdo. 50727, 28080 Madrid, Spain
}

\section{Description}

Superluminal motion in extragalactic jets is successfully explained assuming a relativistic flow of material in a direction close to the observer's line of sight. In rectilinear jets, the apparent velocity measured by the observer $\left(\beta_{a p p}\right)$ is related with the intrinsic velocity of the moving component $(\beta)$ and the angle between the jet direction and the observer's line of sight $(\theta)$ through the equation (eg. Rees 1966, Nature 211, p.468):

$$
\beta_{\text {app }}=\frac{\beta \cdot \sin \theta}{1-\beta \cdot \cos \theta}
$$

We generalize Eq. 1 for knots travelling along arc of circumferences, given that the trajectory and the line of sight lie in the same plane, i.e. the position angle is constant (Fig.1; Lara \& Alberdi, in preparation):

$$
\beta_{a p p}=\frac{\beta \cdot \sin \frac{\Delta \theta_{i j}}{2} \cdot \sin \left(\theta_{i}+\frac{\Delta \theta_{i j}}{2}\right)}{\frac{\Delta \theta_{i j}}{2}-\beta \cdot \sin \frac{\Delta \theta_{i j}}{2} \cdot \cos \left(\theta_{i}+\frac{\Delta \theta_{i j}}{2}\right)}
$$

For a component travelling along a curved path, we can measure not only its apparent velocity, but also its acceleration or deceleration, the variations of its flux density $(S)$, assumed to be produced by changes in the Doppler factor, and possible variations of the spectral index $\left(\alpha ; S \propto \nu^{\alpha}\right)$. This information, altogether, allows us to obtain the intrinsic velocity of the component and the orientation angle with respect to the observer. Provided 


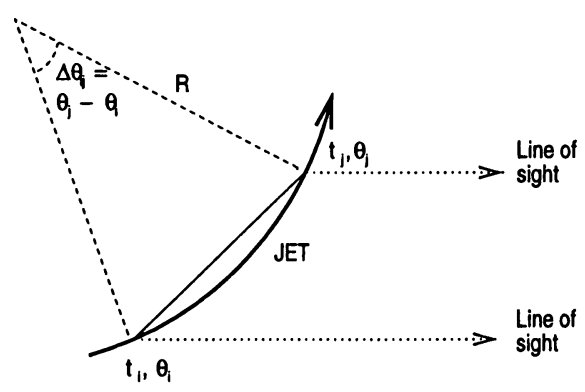

Figure 1. A curved jet observed at two epochs, $t_{i}$ and $t_{j}$

three epochs of observation, $t_{1}, t_{2}$ and $t_{3}$, and assuming that $\beta$ remains constant, we construct a set of equations derived from Eq. 2 and from the relation between the observed and the intrinsic flux density through the Doppler factor $\left(S_{o b s}=S_{i n t r} \cdot D^{3-\alpha}\right.$, for optically thin components):

$$
\begin{gathered}
\beta=\frac{\beta_{a p p} \cdot \frac{\Delta \theta_{12}}{2}}{\sin \frac{\Delta \theta_{12}}{2}\left[\cos \left(\theta+\frac{\Delta \theta_{12}}{2}\right) \cdot \beta_{a p p}+\sin \left(\theta_{1}+\frac{\Delta \theta_{12}}{2}\right)\right]} \\
\Delta \beta_{a p p}=\beta_{a p p}\left(\beta, \theta_{2}, \Delta \theta_{23}\right)-\beta_{a p p}\left(\beta, \theta_{1}, \Delta \theta_{12}\right) \\
\Delta \theta_{i j}=\arccos \left\{\frac{1}{\beta}-\frac{1}{\beta} \cdot\left(1-\beta \cos \theta_{i}\right)^{\frac{3-\alpha_{i}}{3-\alpha_{j}}} \cdot\left[\frac{S_{i}\left(\nu_{i}\right) \nu_{j}^{\alpha_{j}}}{S_{j}\left(\nu_{j}\right) \nu_{j}^{\alpha_{j}}}\right]^{\frac{1}{3-\alpha_{j}}} \cdot \gamma^{\frac{\alpha_{j}-\alpha_{i}}{3-\alpha_{j}}}\right\}-\theta_{i}
\end{gathered}
$$

The spectral index can account for opacity dependent variations in the flux density, although we do not consider the difficulties derived from timeshifts of the turnover frequency which could turn into optically thick the spectrum of the component at a given frequency. After an iterative process we can determine the unknown parameters $\beta, \theta_{1}, \theta_{2}$ and $\theta_{3}$.

\section{Application to $4 \mathrm{C} 39.25$}

The quasar $4 \mathrm{C} 39.25$ shows a superluminal component, usually called $b$, travelling with a non-uniform speed and approximately constant position angle between two stationary ones, and gradually increasing its flux density from 1986 on. Applying our analysis to the available experimental data (Alberdi et al. 1993, ApJ 402, p.160; Alberdi et al. 1995, in preparation), we obtain very consistent results for successive epochs, indicating that the orientation of $b$ was $\sim 9^{\circ}$ in 1984 , getting gradually closer to the line of sight. We derive an intrinsic velocity of $0.974 \mathrm{c}$. These results imply that component $b$ has an intrinsic flux density of $\sim 2.4 \mathrm{mJy}$, and that its deprojected distance to the core is $\sim 40 \mathrm{pc}\left(\mathrm{H}_{o}=100 \mathrm{~km} \mathrm{~s}^{-1} \mathrm{Mpc}^{-1} ; \mathrm{q}_{o}=0.5\right)$. 